TITLE:

\title{
Optimal Subgrid Connection for Space-Time Finite Integration Technique
}

$\operatorname{AUTHOR}(S):$

Sakata, Y.; Mifune, T.; Matsuo, T.

CITATION:

Sakata, Y.... [et al]. Optimal Subgrid Connection for Space-Time Finite Integration Technique. IEEE Transactions on Magnetics 2017, 53(6): 7202304.

ISSUE DATE:

2017-06

URL:

http://hdl.handle.net/2433/243798

\section{RIGHT:}

(C) 2017 IEEE. Personal use of this material is permitted. Permission from IEEE must be obtained for all other uses, in any current or future media, including reprinting/republishing this material for advertising or promotional purposes, creating new collective works, for resale or redistribution to servers or lists, or reuse of any copyrighted component of this work in other works.; この論文は出版社版でありません。引用の際には出版社版をご確認ご利用ください。;This is not the published version. Please cite only the published version. 


\title{
Optimal Subgrid Connection for Space-Time Finite Integration Technique
}

\author{
Y. Sakata ${ }^{1}$, T. Mifune ${ }^{1}$, Member, IEEE, and T. Matsuo ${ }^{1}$, Member, IEEE \\ ${ }^{1}$ Graduate School of Engineering, Kyoto University, Kyoto 615-8510, Japan
}

The subgrid connection for the space-time finite integration technique is optimized based on the propagation condition of plane waves. This condition results in an algebraic equation determining the optimization parameters for the grid connection. $2 \mathrm{D}$ wave propagation simulations show that this method generates optimal subgrid connections that reduce unphysical wave reflections.

Index Terms_Finite integration technique (FIT), optimization, space-time grid, subgrid, unphysical wave reflection

\section{INTRODUCTION}

$\mathrm{T}$ HE SUBGRID technique [1], [2] achieves a local grid refinement for the FDTD method. Its nonconformal grid connection, however, often induces numerical instability and unphysical wave reflections. To overcome these difficulties, higher-order schemes [3], [4] and filtering methods [4], [5] are sometimes introduced at some expense to computation times. The finite integration technique (FIT) [6] is an option to realize a stable subgrid scheme. Recently, the space-time FIT [7], [8] has been developed and achieves a local space-time grid refinement without inducing instability [9], [10]. Using no interpolation or filtering method for subgrid connection, the space-time FIT reduces unphysical wave reflections compared with the spatial FIT [11].

To date, higher-order schemes and variable interpolations have not been used in the space-time FIT because they may undermine the stability of numerical solutions. Instead, this paper attempts to improve the numerical accuracy by grid optimization at the subgrid connection. A general method for grid optimization is presented based on a condition for planewave propagation.

\section{SPACE-Time Finite InTEgRATION TEChNiQUe}

The Maxwell equations without source term is written in integral form as [7]

$$
\begin{gathered}
\oint_{\partial \Omega_{p}} F=0, \oint_{\partial \Omega_{d}} G=0, \\
F=-\sum_{i=1}^{3} E_{i} \mathrm{~d} t \mathrm{~d} x^{i}+\sum_{j=1}^{3} B_{j} \mathrm{~d} x^{k} \mathrm{~d} x^{l}, \\
G=\sum_{i=1}^{3} H \mathrm{~d} t \mathrm{~d} x^{i}+\sum_{j=1}^{3} D_{j} \mathrm{~d} x^{k} \mathrm{~d} x^{l},
\end{gathered}
$$

where $(j, k, l)$ is a cyclic permutation of $(1,2,3) ; \Omega_{p}$ and $\Omega_{d}$ are hypersurfaces in space-time. The electromagnetic variables in the FIT are defined as

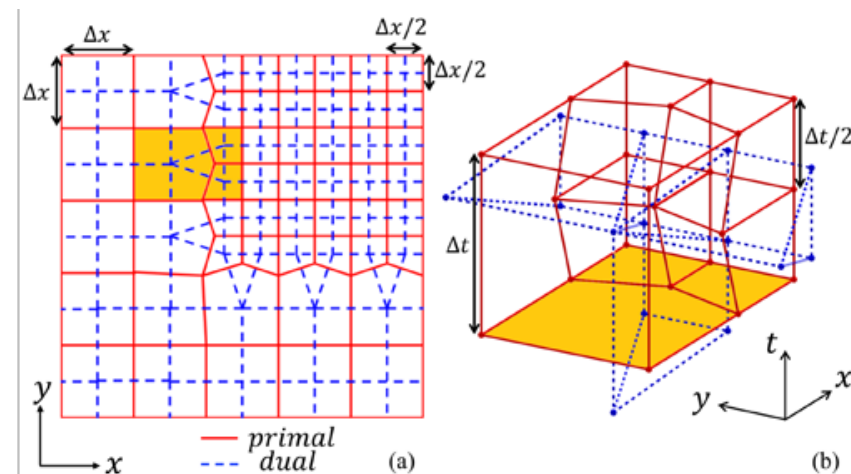

Fig. 1. Space-time subgrid; (a) Spatially local grid refinement and (b) spacetime connection between main grid and subgrid.

$$
f=\int_{S_{p}} F, g=\int_{S_{d}} G
$$

where $S_{p}$ and $S_{d}$ are the faces of primal and dual grids that compose $\partial \Omega_{p}$ and $\partial \Omega_{d}$. For simple expressions of the constitutive equation, the Hodge dual grid [7] is used,

$$
\frac{\int_{S_{d}} c \mathrm{~d} t \mathrm{~d} x^{j}}{\int_{S_{p}} \mathrm{~d} x^{k} \mathrm{~d} x^{l}}=-\frac{\int_{S_{d}} \mathrm{~d} x^{k} \mathrm{~d} x^{l}}{\int_{S_{p}} c \mathrm{~d} t \mathrm{~d} x^{j}}=\kappa,
$$

where $c=1 / \sqrt{\varepsilon \mu}$ and $\varepsilon$ and $\mu$ are the permittivity and permeability, respectively. From (4) and (5), $f=Z g / \kappa$, where $Z=\sqrt{\mu / \varepsilon}$ is the impedance.

Fig. 1 illustrates a simple space-time subgrid [9], where the main grid has spatial grid-size $\Delta x$ and temporal step $\Delta t$, which are divided into half-size on the subgrid. The main grid and subgrid are connected so that the primal grid is orthogonal to its dual grid with respect to the Lorentzian metric. Nonorthogonality at corners of the subgrid connection may cause numerical error including unphysical wave reflections. This can be improved using the vectorial symmetric correction [10], [11] but is not discussed in this paper.

\section{OPTIMIZATION OF 2D SPATIAL SUbGRID CONNECTION}

First, a 2D spatial subgrid connection is optimized. The faces and edges can be curved [Fig. 2] without changing node positions because the variables are defined in integral form (4). The connection between the main grid and subgrid is optimized by changing face areas of the grids. 


\section{A. Propagation Condition of Plane Wave}

The propagation of a plane wave $\left(E_{x}, E_{y}, B_{z}\right)$ is examined:

$$
\begin{aligned}
& E_{x}=E_{x a} \exp \left[j\left(\omega t-k_{x} x-k_{y} y\right)\right], \\
& E_{y}=E_{y a} \exp \left[j\left(\omega t-k_{x} x-k_{y} y\right)\right], \\
& B=B_{a} \exp \left[j\left(\omega t-k_{x} x-k_{y} y\right)\right],
\end{aligned}
$$

where $\omega$ is the angular frequency and $\boldsymbol{k}=\left(k_{x}, k_{y}\right)$ is the wavenumber vector. For simplicity, the speed of light $c$ (= $\left.\omega / \sqrt{k_{x}^{2}+k_{y}^{2}}\right)$ and the impedance $Z\left(=\sqrt{E_{x}^{2}+E_{y}^{2}} / H\right)$ are set to unity by normalization. Thereby

$$
\begin{aligned}
& k_{x}=\omega \cos \theta, k_{y}=\omega \sin \theta, \\
& E_{y a}=B_{a} \cos \theta, E_{x a}=-B_{a} \sin \theta,
\end{aligned}
$$

where $\theta$ is the azimuthal angle of the wavenumber vector.

The variables are located on the primal grid [Fig. 2(b)], where $\delta$ is a free parameter for the node positions at the primal grid connection. Variables $b$ and $e$ are defined as $f$ in (4). Corresponding to them, $h$ and $d$ are given as $g$ in (4) on the dual grid. The substitution of (6) into the time-marching scheme results in

$$
\begin{aligned}
& d_{x 0} \Delta_{\text {time }}=h_{0} \Delta_{\text {space }} \\
& d_{x 10} \Delta_{\text {time }}=h_{10}-h_{11} \exp \left(j k_{y} \Delta x\right) \\
& d_{x 11} \Delta_{\text {time }}=h_{11}-h_{10} \\
& d_{y 1 i} \Delta_{\text {time }}=-\left(h_{1 i}-h_{0}\right), \quad(i=0,1) \\
& b_{0} \Delta_{\text {time }}=-\left(e_{y 10}+e_{y 11}-e_{y 0}\right)+e_{x 0} \Delta_{\text {space }} \\
& b_{10} \Delta_{\text {time }}=-\left(e_{y 20}-e_{y 10}\right)+\left(e_{x 11}-e_{x 10}\right) \\
& b_{11} \Delta_{\text {time }}=-\left(e_{y 21}-e_{y 11}\right) \\
& \quad+\left[e_{x 10} \exp \left(-j k_{y} \Delta x\right)-e_{x 11}\right]
\end{aligned}
$$

where $\Delta_{\text {time }}$ and $\Delta_{\text {space }}$ are set as

$$
\begin{aligned}
& \Delta_{\text {time }}=\exp (j \omega \Delta t / 2)-\exp (-j \omega \Delta t / 2), \\
& \Delta_{\text {space }}=\exp \left(-j k_{y} \Delta x / 2\right)-\exp \left(j k_{y} \Delta x / 2\right) .
\end{aligned}
$$

The constitutive equations are written as

$$
\begin{aligned}
& d_{x 0}=\frac{e_{x 0}}{\Delta t(1-\delta)}, d_{x 10}=\frac{e_{x 10}}{2 \Delta t\left(\frac{1}{2}+\delta\right)}, \\
& d_{x 11}=\frac{e_{x 11}}{2 \Delta t\left(\frac{1}{3}+\delta\right)}, d_{y 1 i}=\frac{3 e_{y 1 i}}{2 \Delta t}, d_{y 2 i}=\frac{e_{y 2 i}}{\Delta t}, \\
& h_{0}=\frac{\Delta t b_{0}}{S_{B C}}, h_{1 i}=\frac{\Delta t b_{1 i}}{S_{B F}}, \quad(i=0,1), \\
& S_{B C}=\Delta x^{2}(1-2 p), S_{B F}=\Delta x^{2}(1 / 4+p),
\end{aligned}
$$

where $p$ is a free parameter. The relations (6) and (7) provide

$$
\begin{aligned}
& e_{y 0}=\frac{\Delta x \Delta t(\cos \theta) b_{0} \exp \left(\frac{j k_{x} \Delta x}{2}\right)}{S_{B C}}, \\
& e_{y 2 i}=\frac{\Delta x \Delta t(\cos \theta) b_{1 i} \exp \left(-\frac{j k_{x} \Delta x}{4}\right)}{2 S_{B F}}, \quad(i=0,1) .
\end{aligned}
$$

Equations (8)-(17) are rewritten as

$$
\frac{e_{x 10} \Delta_{\text {time }}}{2 \Delta t\left(\frac{1}{2}+\delta\right)}-\frac{\Delta t\left[b_{10}-b_{11} \exp (j \omega \Delta x \sin \theta)\right]}{S_{B F}}=0 \text {, }
$$

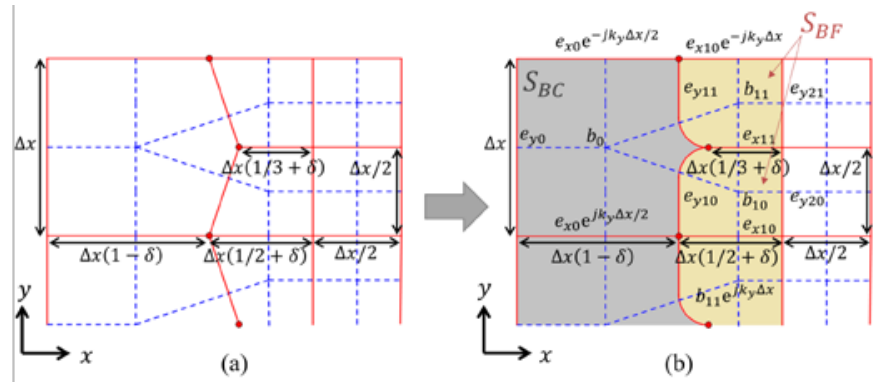

Fig. 2. Subgrid connection; (a) spatial connection and (b) variables allocated to primal grid, where $S_{B C}=\Delta x^{2}(1-2 p)$ and $S_{B F}=\Delta x^{2}(1 / 4+p)$ are areas of gray and yellow faces, respectively.

$$
\begin{aligned}
& \frac{e_{x 11} \Delta_{\text {time }}}{2 \Delta t\left(\frac{1}{3}+\delta\right)}-\frac{\Delta t\left(b_{11}-b_{10}\right)}{S_{B F}}=0, \\
& \frac{3 e_{y 1 i} \Delta_{\text {time }}}{2 \Delta t}+\frac{\Delta t b_{10}}{S_{B F}}-\frac{\Delta t b_{0}}{S_{B C}}=0, \quad(i=0,1), \\
& b_{0} \Delta_{\text {time }}-\frac{\Delta x \Delta t(\cos \theta) b_{0} \exp \left(\frac{j \omega \Delta x \cos \theta}{2}\right)}{S_{B C}} \\
& +\frac{\Delta x \Delta t(1-\delta)(\sin \theta) b_{0} \Delta_{\text {space }}}{S_{B C}}+e_{y 10}+e_{y 11}=0, \\
& b_{10} \Delta_{t i m e}+\frac{\Delta x \Delta t(\cos \theta) b_{10} \exp \left(-\frac{j \omega \Delta x \cos \theta}{4}\right)}{2 S_{B F}}-e_{y 10}-e_{x 11}+e_{x 10}=0, \\
& b_{11} \Delta_{t i m e}+\frac{\Delta x \Delta t(\cos \theta) b_{11} \exp \left(-\frac{j \omega \Delta x \cos \theta}{4}\right)}{2 S_{B F}} \\
& -e_{y 11}-e_{x 10} \exp (-j \omega \Delta x \sin \theta)+e_{x 11}=0 .
\end{aligned}
$$

Defining the variable vector

$$
\boldsymbol{V}_{1}=\left(b_{0}, b_{10}, b_{11}, e_{x 10}, e_{x 11}, e_{y 10}, e_{y 11}\right)^{\mathrm{T}} \text {, }
$$

(18)-(23) are rewritten as

$$
\mathrm{A}_{1} \boldsymbol{V}_{1}=\mathbf{0} \text {, }
$$

where $A_{1}$ is a constant matrix. The propagation of the plane wave requires that vector $\boldsymbol{V}_{1}$ is nonzero, which means that

$$
\operatorname{det}\left(\mathrm{A}_{1}\right)(\omega, \theta, \delta, p)=0 .
$$

It is impossible to satisfy (26) completely for arbitrary $\omega$ and $\theta$. It suffices that (26) is approximately satisfied when the grid size is sufficiently small compared with the wavelength, in other words, when $\omega$ is sufficiently small. Consequently, after $\operatorname{det}\left(\mathrm{A}_{1}\right)$ is Maclaurin-expanded with respect to $\omega$, it is approximated by the term having the lowest order of $\omega$. Using

$$
\exp (z) \approx \sum_{n=0}^{l}\left(z^{n} / n !\right),
$$

with $l=4$, $\operatorname{det}\left(\mathrm{A}_{1}\right)$ is approximated as

$$
\begin{aligned}
& \operatorname{det}\left(\mathrm{A}_{1}\right)(\omega, \theta, \delta, p) \approx \\
& \frac{9(\cos \theta) \Delta t^{3}[(48 \delta-5) \cos \theta+96 p-48 \delta+2] \omega^{4}}{2(3 \delta-1)(2 p-1)(4 p+1)^{2}}
\end{aligned}
$$

where $\Delta x$ is set to 1 by normalization for simplicity. To eliminate this for arbitrary $\theta$, the parameters $\delta$ and $p$ are optimized,

$$
\delta=\frac{5}{48}, p=\frac{1}{32} .
$$

It is expected that the parameters determined from the plane- 

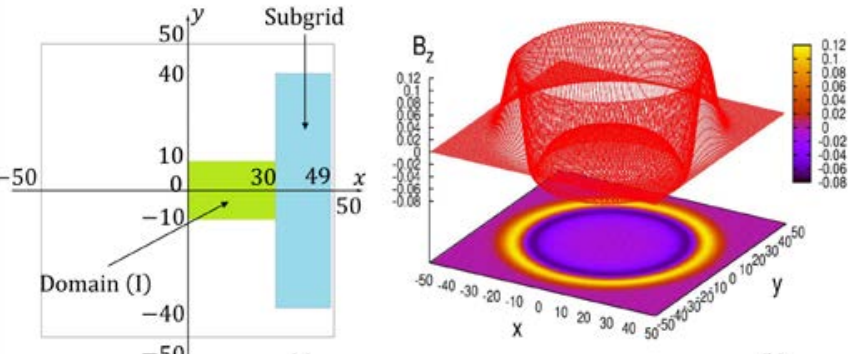

(a)

(b)

Fig. 3. Wave propagation simulation; (a) analyzed domain and (b) distribution of $B_{z}$ at $x^{0}=105 \Delta t$ with $\Delta t=0.35$.

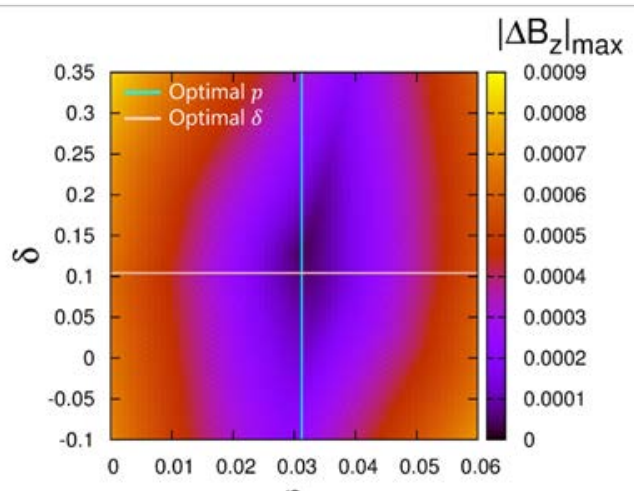

$\mathrm{p}$

Fig. 4. Average discrepancy $\left|\Delta B_{z}\right|_{\text {max }}$ depending on parameters $p$ and $\delta$.

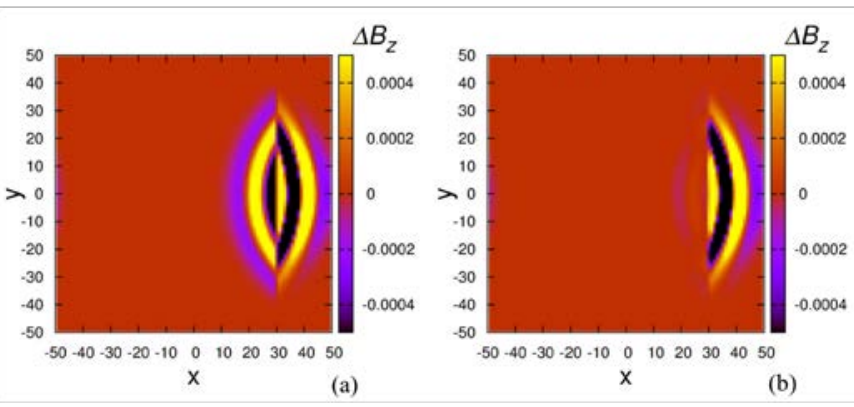

Fig. 5. Distribution of discrepancy $\Delta B_{z}$ between the FDTD method and the FIT; (a) w/o optimization, (b) w/ optimization.

wave condition above improve the accuracy of discretization and consequently suppress the unphysical wave reflection.

\section{B. Numerical examination}

For simplicity, the spatial step $\Delta x$ and the speed of light $c$ are set to unity by normalization. An electromagnetic field is analyzed in $[-50: 50] \times[-50: 50]$ including a connecting spatial subgrid $[30: 49] \times[-40: 40] \quad[$ Fig. 3(a)]. The normalized initial condition is given by $E_{x}=E_{y}=0$ and $B_{z}=$ $\left.\exp \left[-\left(x^{2}+y^{2}\right)\right] / 25\right]$. The distribution of $B_{z}$ at $t=105 \Delta t$ with $\Delta t=0.35$ is illustrated in Fig. 3(b).

Fig. 4 depicts the maximal magnitude of the unphysical wave reflection $\left|\Delta B_{z}\right|_{\text {max }}$ which depends on $\delta$ and $p$ in domain (I) $[0: 30] \times[-10: 10]$ [Fig. 3(a)]. The optimal condition (29) agrees with the minimal point of $\left|\Delta B_{z}\right|_{\text {max }}$ in Fig. 4. Fig. 5 shows the discrepancy in $B_{Z}$ between that obtained using the FDTD method and that from the FIT with an optimal spatial subgrid for $t=105 \Delta t$ with $\Delta t=0.35$. The discrepancy in the domain $x>30$ is mainly a consequence of numerical dispersion whereas the discrepancy in the domain $x<30$ is from unphysical wave reflections. The proposed optimization method has effectively reduced the unphysical wave reflections.

\section{3D SPACE-TIME Optimal SUBgrid}

The optimization of subgrid connection based on the propagation condition of the plane wave is applied to the 3D space-time subgrid with a 2D space. The spatial 2D electromagnetic field consisting of $\left(E_{x}, E_{y}, B_{z}\right)$ is described on the faces of a primal grid $S_{p}$ and the edges of a dual grid $l_{d}$ whereas the $\left(B_{x}, B_{y}, E_{z}\right)$ mode analysis uses the edges of a primal grid $l_{p}$ and the faces of a dual grid $S_{d}$.

\section{A. $\left(E_{x}, E_{y}, B_{z}\right)$ Mode}

Space-time variables allocated on the primal grid are illustrated in Fig. 6. The spatial and temporal face areas $S_{B C}, S_{B F}, S_{E C}$ and $S_{E F}$ are given as

$$
\begin{aligned}
& S_{B C}=\Delta x^{2}(1-2 p), S_{B F}=\Delta x^{2}(1 / 4+p), \\
& S_{E C}=\Delta x \Delta t(1-2 r), S_{E F}=\Delta x \Delta t(1 / 4+r),
\end{aligned}
$$

where $p$ and $r$ are free parameters. These face areas are optimized in accordance with the propagation condition of the plane wave. For simplicity, $\Delta x$ is set to 1 hereafter.

The substitution of (6) into the time-marching scheme leads to the propagation condition denoted as

$$
\begin{aligned}
& \mathrm{A}_{2} \boldsymbol{V}_{2}=\mathbf{0}, \\
& \boldsymbol{V}_{2}=\left(\boldsymbol{b}, \boldsymbol{e}_{x}, \boldsymbol{e}_{y}\right)^{\mathrm{T}}, \boldsymbol{b}=\left[b_{0}, b_{10}^{0}, b_{11}^{0}, b_{10}^{1}, b_{11}^{1}\right], \\
& \boldsymbol{e}_{\boldsymbol{x}}=\left[e_{x 10}^{0}, e_{x 11}^{0}, e_{x 10}^{1}, e_{x 11}^{1}\right], \\
& \boldsymbol{e}_{\boldsymbol{y}}=\left[e_{y 10}^{0}, e_{y 11}^{0}, e_{y 10}^{1}, e_{y 11}^{1}, e_{y 20}^{0}, e_{y 21}^{0}, e_{y 20}^{1}, e_{y 21}^{1}\right] .
\end{aligned}
$$

Using (27) with $l=6$, the propagation condition is written as

$$
\begin{array}{r}
\operatorname{det}\left(\mathrm{A}_{2}\right)(\omega, \theta, p, r, \Delta t)=\left[(144 r-8) \cos ^{2} \theta\right. \\
\left.+5 \Delta t^{2}+144 p-144 r+3\right] C_{0} \omega^{6} \\
+O\left(\omega^{7}\right)=0,
\end{array}
$$

where $C_{0}$ is a non-zero constant. To eliminate the coefficient of $\omega^{6}$, the optimal condition for arbitrary angle $\theta$ is given as

$$
p=\frac{5\left(1-\Delta t^{2}\right)}{144}, r=\frac{1}{18} \text {. }
$$

This condition was verified in a numerical simulation on the domain [Fig. 3(a)]. Fig. 7(a) depicts the maximal magnitude of the unphysical wave reflection $\left|\Delta B_{z}\right|_{\max }$, which depends on $p$ and $r$. Similar to the optimization of the 2D spatial subgrid connection, the best parameters giving the minimal $\left|\Delta B_{z}\right|_{\text {max }}$ are predicted by the optimization. Fig. 7(b) shows unphysical wave reflections along $y=0$ when $t=90 \Delta t$ with $\Delta t=0.4$, where the optimal parameters reduce the reflection.

\section{B. $\left(B_{x}, B_{y}, E_{z}\right)$ Mode}

This section discusses a field having components $\left(B_{x}, B_{y}, E_{z}\right)$. The primal grid connection is optimized using parameter $\delta$ (Fig. 8) whereas the dual grid is fixed.

Variables for the $\left(B_{x}, B_{y}, E_{z}\right)$ mode are allocated on the primal grid (Fig. 8). The equation of propagation for the plane wave is written

$$
\mathrm{A}_{3} \boldsymbol{V}_{3}=\mathbf{0} \text {, }
$$



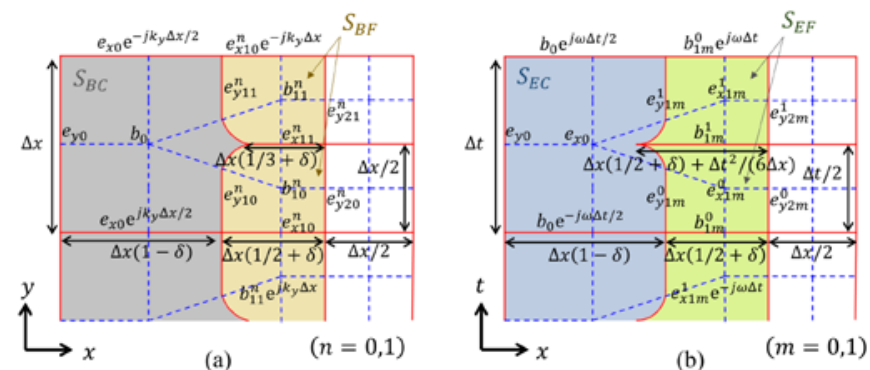

Fig. 6. Assignment of variables for wave of $\left(E_{x}, E_{y}, B_{z}\right)$ to 3D space-time subgrid with 2D space; (a) spatial grid, (b) space-time grid.
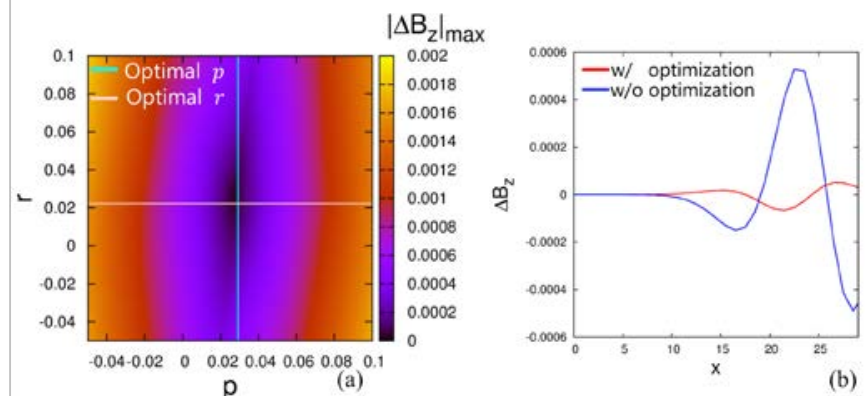

(b)

Fig. 7. (a) Maximal magnitude $\left|\Delta B_{z}\right|_{\text {max }}$ depending on $p$ and $r$, (b) unphysical wave reflection with and without optimization.

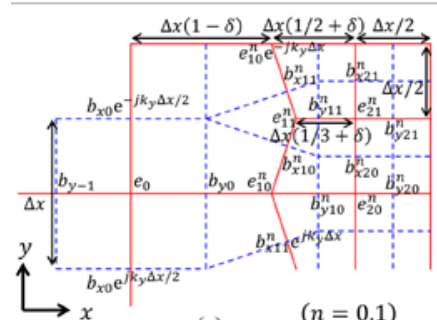

(a)

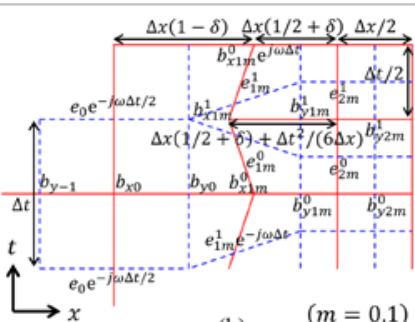

(b) $\quad(m=0,1)$
Fig. 8. Allocation of electromagnetic field variables consisting of $\left(B_{x}, B_{y}, E_{z}\right)$; (a) spatial grid and (b) space-time grid.

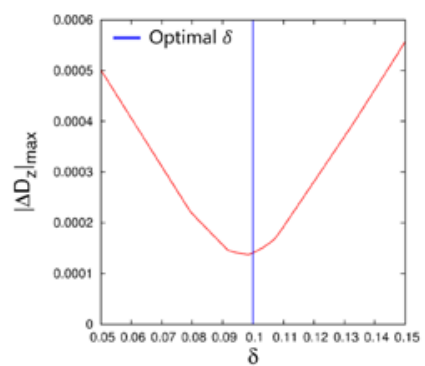

(a)

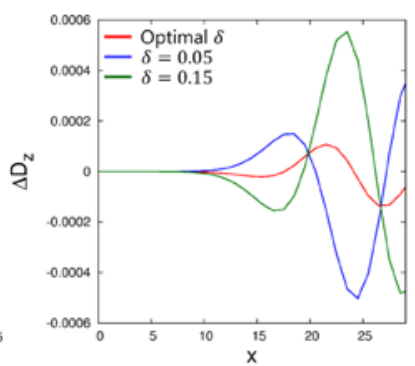

(b)
Fig. 9. (a) Distribution of the maximal magnitude $\left|\Delta D_{z}\right|_{\text {max }}$ depending on $\delta$, (b) unphysical wave reflection with $\delta=0.05,0.15$ and optimal $\delta$.

$$
\begin{aligned}
& \boldsymbol{V}_{3}=\left(\boldsymbol{e}, \boldsymbol{b}_{x}, \boldsymbol{b}_{y}\right)^{\mathrm{T}}, \boldsymbol{b}_{\boldsymbol{y}}=\left[b_{y 10}^{0}, b_{y 11}^{0}, b_{y 10}^{1}, b_{y 11}^{1}\right], \\
& \boldsymbol{b}_{\boldsymbol{x}}=\left[b_{x 10}^{0}, b_{x 11}^{0}, b_{x 10}^{1}, b_{x 11}^{1}, b_{x 20}^{0}, b_{x 21}^{0}, b_{x 20}^{1}, b_{x 21}^{1}\right], \\
& \boldsymbol{e}=\left[e_{0}, e_{10}^{0}, e_{11}^{0}, e_{10}^{1}, e_{11}^{1}, e_{20}^{0}, e_{21}^{0}, e_{20}^{1}, e_{21}^{1}\right] .
\end{aligned}
$$

The propagation condition is given as

$$
\begin{gathered}
\operatorname{det}\left(\mathrm{A}_{3}\right)(\omega, \theta, \delta, \Delta t)=\left[\left(3 \Delta t^{2}+72 \delta-12\right) \cos ^{4} \theta\right. \\
\left.+\left(\Delta t^{2}+5\right) \cos ^{2} \theta+\Delta t^{2}-1\right] C_{1} \omega^{6} \\
+O\left(\omega^{7}\right)=0,
\end{gathered}
$$

where $C_{1}$ is a nonzero constant. From (37), the optimal condition for $\theta=0$ yields

$$
\delta=\frac{8-5 \Delta t^{2}}{72} .
$$

Wave propagation is simulated to examine the accuracy of condition (38) on the domain [Fig. 3(a)]. The normalized initial conditions are $B_{x}=B_{y}=0, E_{z}=\exp \left[-\left(x^{2}+y^{2}\right) / 25\right]$. Fig. 9(a) plots the maximum of $\left|\Delta D_{z}\right|$ measured in domain (I) [Fig. 3(b)]. Condition (38) provides the optimal value of $\delta$ that suppresses the unphysical wave reflection. Fig. 9(b) gives the distribution of the discrepancy in $\Delta D_{z}$ between that obtained using the FDTD method and that from the space-time FIT along $y=0$ at $t=90 \Delta t$ with $\Delta t=0.4$, where the optimal parameter reduces reflections. A further reduction of unphysical reflections requires the optimization of the dual grid.

\section{CONCLUSION}

Using the propagation condition of plane waves, the subgrid construction for the space-time FIT has been optimized to reduce unphysical wave reflections. A simple optimization of the 4D space-time grid is ensued from an analysis that decomposes the field into $\left(E_{x}, E_{y}, B_{z}\right)$ and $\left(B_{x}, B_{y}, E_{z}\right)$ modes discussed in this paper.

\section{REFERENCES}

[1] A. Taflove and S. C. Hagness, Computational Electrodynamics, The Finite Difference Time Domain Method, 3rd ed., Artech House, Boston, 2005.

[2] Y. Hao and R. Mittra, FDTD Modeling of Metamaterials: Theory and Applications, Artech House, 2009.

[3] M. Okoniewski, E. Okoniewska, and M. A. Stuchly, "Three-dimensional subgridding algorithm for FDTD,” IEEE Trans. Antennas and Propaga., vol. 45, no. 3, pp. 422-429, Mar. 1997.

[4] T. Ohtani, Y. Kanai, and N. V. Kantartzis, "A 4-D subgrid scheme for the NS-FDTD technique using the CNS-FDTD algorithm with the Shepard method and a Gaussian smoothing filter,” IEEE Trans. Magn., vol. 51, no. 3, 7201004, Mar. 2015.

[5] A. Vaccari, R. Pontalti, C. Malacarne, and L. Cristoforetti, “A robust and efficient subgridding algorithm for finite-difference time-domain simulations of Maxwell's equations,” J. Comp. Phys., vol. 194, no. 1, pp. 117-139, Feb. 2004.

[6] P. Thoma and T. Weiland, "A consistent subgridding scheme for the finite difference time domain method,” Int. J. Numer. Model., vol. 9, no. 5, pp. 359-374, Sept. 1996.

[7] T. Matsuo, "Space-time finite integration method for electromagnetic field computation,” IEEE Trans. Magn., vol. 47, no. 5, pp. 1530-1533, May 2011.

[8] J. Kawahara, T. Mifune, and T. Matsuo, "Geometrical formulation of a 3D space-time finite integration method," IEEE Trans. Magn., vol. 49, pp. 1693-1696, May 2013.

[9] T. Matsuo, T. Shimoi, J. Kawahara, and T. Mifune, “A simple sub-grid scheme using space-time finite integration method," IEEE Trans. Magn., vol. 51, no. 3, 7201904, Mar. 2015.

[10] T. Matsuo, J.Kawahara, T. Shimoi, and T.Mifune, "Stability analysis of space-time finite integration schemes,” Compel, vol. 34, no. 5, pp. 16091622, Sep. 2015.

[11] K. Arai, T. Mifune, and T. Matsuo, "Space-time PML and subgrid connections for finite integration method,” IEEE Trans. Magn., vol. 52, no. 3, 7206004, Mar. 2016. 